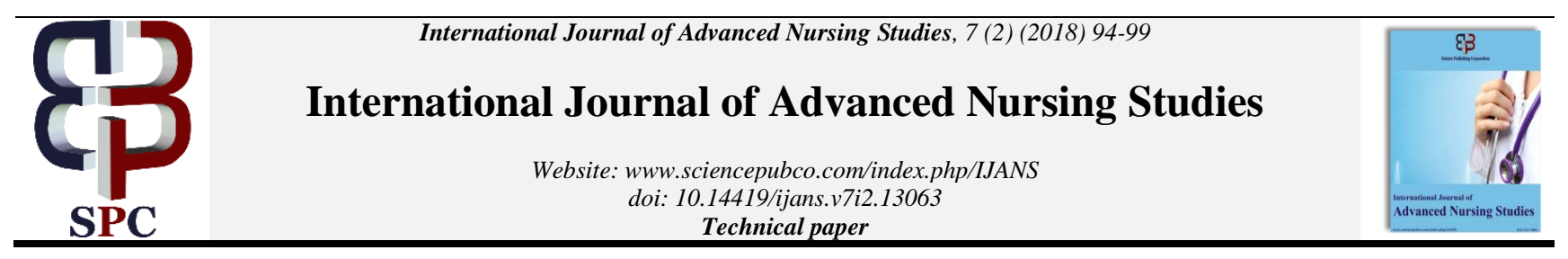

\title{
Effect of designed nursing instructions on knowledge and self-care behavior among patients with chemotherapy induced neutropenia
}

\author{
Asmaa Sayed Abd-Almageed * \\ Lecturer of Medical Surgical Nursing, Faculty of Nursing, Assist University \\ *Corresponding author E-mail:
}

\begin{abstract}
Background:

Chemotherapy-induced neutropenia (CIN) is the main dose limiting toxicity of systemic chemotherapy and it is associated with significant morbidity, mortality and treatment cost.

Aim:

Of the study was to evaluate effect of designed nursing instructions on knowledge and self-care behavior among patients with chemotherapy-induced neutropenia.

Research design: Pre-post research design was utilized.

Setting: The study was conducted at internal Medical Oncology Department at South Egypt Cancer Institute.

Sample: Sixty adult patients with chemotherapy induced neutropenia. Tools of data collection: Tool (I): Patient assessment sheet, Tool (II): Self-care behavior assessment.

Results: There was a highly statistically significant difference between pre and post applying of designed nursing instructions as regard patients' level of knowledge and self- care behavior of the studied patients $(\mathrm{p}<0.01)$.

Conclusion: The designed nursing instructions improved knowledge and self-care behavior among patients with chemotherapy-induced neutropenia.

Recommendation: Each neutropenic patient should be given a booklet including knowledge about neutropenia, importance, and how to practice standards self-care behavior. Education of patients and families are keys to prompt recognition about potentially life-threatening symptoms associated with neutropenia.
\end{abstract}

Keywords: Chemotherapy Induced Neutropenia; Designed-Nursing Instructions; Self-Care Behavior.

\section{Introduction}

Patients undergoing myelo-suppressive chemotherapy are at risk of developing neutropenia which defined as an absolute neutrophil count below 500/mm3, a matter that may lead to life-threatening infections which may quickly lead to sepsis, and death (Hawley et al., 2011). Severe neutropenia and febrile neutropenia (FN) are therefore major causes of morbidity, treatment interruptions and dose reductions in patients undergoing chemotherapy. (Weycker et al., 2014)

Neutropenia itself does not cause any symptoms, often it is spotted during routine blood tests or tests for another condition. For this reason and others, patients undergoing chemotherapy who are most at risk from the condition must have regular blood tests. (Akhil, 2014)

Due to the type and intensity of treatment received and other risk factors, many cancer patients experience a decrease in elements of the immune system that make them more exposed to various infections. Neutropenia is considered as an oncology emergency and can lead to severe adverse consequences such as serious infection complications and death (Villafuerte et al., 2014).Nurses are therefore have avery important role in identifying patients at risk for devel- oping neutropenia and monitor patients who already have it for better initiation of interventions to provide high quality care for patients receiving chemotherapy for cancer treatment. (Ropka and $\mathrm{Pa}$ dilla, 2007)

Self-care behavior is that a patient accepts to care for himself to promote health and well-being. It provides patients with a greater sense of security, and the knowledge necessary to ensure that health is well-preserved as best as possible (Artinian et al., 2002 and Jaarsma, 2008). Patient and visitor education is an essential aspect of the care of neutropenic patients; part of that education process should be the provision of written information to reinforce any verbal information. Patient education is the process by which health professionals and others impart information to patients and their caregivers that will alter their health behaviors or improve their health status, Patients who recognize that nurses are providing education and support in self-care are more likely to respond when patients realize the benefits of self-care. (Bastable et al., 2011)

Oncology nurses play a vital role in the education of patients and caregivers concerning neutropenia precautions. Patients will need to know how to avoid infection, what the signs of infection are, and when to seek medical care. They should be instructed to wash hands frequently, avoid crowds or anyone who is sick with a cold or flu. Avoid cleaning up pet excrement, bath daily, brush teeth twice a day. Avoid constipation by regularly taking a stool softener. Avoid 
use of suppositories or enemas. Eat only fruits and vegetables that can be washed or cooked. Eat only meat that is completely cooked. Avoid drinking unpasteurized milk or other beverages. (Loerzel, 2005)

Significance of the study:

According to South Egypt Cancer Institute record in (2015); 2856 cases received chemotherapy, in (2016) 3055 cases and in (2017); the number of patients increased to 4300 cases, but the incidence of neutropenia was not accessible. Also, from the researchers' experiences, it was noted that patients' knowledge and self-care behavior about neutropenia inadequate and need for improvement. Therefore this study is considered the first one in this geographical location to help neutropenic patients to improve their knowledge and self-care behavior.

Aim of the study:

Aim of the study was to evaluate effect of designed nursing instructions on knowledge and self -care behavior among patients with chemotherapy induced neutropenia

Research hypothesis:

Knowledge and self -care behavior among patients with chemotherapy induced neutropenia will be better after application of designed nursing instructions.

\section{Patients and methods}

\subsection{Research design}

Pre /and post research design was utilized to conduct this study. Study variables: The independent variable in this study was the designed nursing instructions. While the dependent variables were the knowledge and self-care behavior among patients with chemotherapy induced neutropenia.

\subsection{Setting}

The study was conducted at internal Medical Oncology Department at South Egypt Cancer Institute.

\subsection{Patients}

A purposive sample of sixty adult neutropenic patients admitted in internal Medical Oncology Department, both male and female, their age ranged between (18-65) years and agreed to participate in the study.

Exclusive criteria:

Patients with visual or hearing impairment, known cognitive impairment, known psychiatric illness, and unable to carry out selfcare activities.

\subsection{Tools of the study two tools were utilized in this study}

Tool (I): Patient assessment sheet:

This sheet was developed by the researcher based on literature review.

It was used pre and post applying the designed nursing instructions to assess the exact knowledge level of patients about neutropenia. It included two parts:

Part 1: patients' demographic and medical data:

- Demographic data including age, sex, marital status, level of education, type of employment, and smoking.

- Medical data include type of cancer, presence of chronic illness, medication use, and (ECOG performance).

\section{ECOG performance:}

It was developed by the Eastern Cooperative Oncology Group Oken et al., (1982). It describes the patient's level of functioning in terms of their ability to care for themself, daily activity, and physical ability, it was contain 6 grades, grade (0) refer to fully active, able to carry on all pre-disease performance without restriction, grade (1) restricted in physically strenuous activity but ambulatory and able to carry out work of a light or sedentary nature, light house work, office work, grade (2) ambulatory and capable of all self- care but unable to carry out any work activities; up and about more than 50\% of waking hours, grade (3) capable of only limited self-care; confined to bed or chair more than $50 \%$ of waking hours, grade (4) completely disabled; cannot carry on any self -care; totally confined to bed or chair and grade (5) dead.

Part 2: knowledge assessment

Knowledge assessment sheet about neutropenia was developed by the researcher based on current national and international literature. It included 12 multiple choice questions regarding (function of white blood cells, symptoms of infection, symptoms of sepsis, items suitable to place inside the bed room for patients receiving chemotherapy, food that should be avoided to prevent infection, measures carried out every day in order to prevent infection, measures that must be taken for patient receiving chemotherapy to stay in a public area, methods to prevent infection, measures to prevent oral mucositis and measures that should be taken if patient has fever).

Scoring system:

Total number of questions was twelve, each question contained five responses (from A to E), and the patient chooses the answer.

The total score of patients' knowledge questionnaire sheet was 60 degrees:

- More than $50 \%$ means satisfactory

- Less than $50 \%$ means unsatisfactory

Tool (II): Self-care behavior assessment, through using (life habits assessment sheet for patients: It was developed by Polit et al., (2007).to assess the exact level of self- care behavior for neutropenic patients. The Parson's correlation coefficient of different sections of the questionnaire ranged from 0.723 to 0.976 which indicated strong to very strong association. The questionnaire used a four-point Likert scale ranging from "Never," "Seldom," "Sometimes," and "Always." The overall behavior would be calculated by adding up all of the responses to 23 questions (i.e., "Never" = one, "Seldom" = [2], "Sometimes" = [3], and "Always" =4). Reversal of score would be given on diet habit (i.e., "Never" = four, "Seldom" = [3], "Sometimes" = [2], and "Always" = 1).

- More than $50 \%$ means Positive self -care behavior.

- Less than $50 \%$ means negative self -care behavior.

The overall behaviors were divided into four categories including personal hygiene, hand hygiene, diet hygiene, and oral hygiene. This tool composed of 23 questions:

Personal hygiene: include [4] items (wear mask in the public area, observe any new wound, take shower every day, and take temperature every day).

Hand hygiene: include 8 items (wash your hands more than twenty seconds each time, wash your hands with soaps, wash hands after using the toilet, wash hands after visiting hospital, wash hands before eating or handling food, wash hands after touching public installations or equipment, such as escalator buttons, wash hands when hands are contaminated by respiratory secretions, e.g. after coughing or sneezing, bring along the alcohol hand rub with you when go out).

Dietary hygiene: include [4] items (eat overnight food, eat uncooked food (E.g. Half-cooked egg), eat salad, and eat rotten fruit). Oral hygiene: include [7] items (mouthwash at least four times per day, mouthwash after wake up, mouthwash after every meal and snack, mouthwash before bed, use soft toothbrush to clean your teeth and tongue, check oral cavity every day, and wash the toothbrush after using it).

The designed nursing instructions:

It was designed to allow the patients to recognize goals and values, understand how patient's behavior influences their health, develop knowledge, skills and confidence to make decisions about their health that best enable them to achieve the goals. The researcher developed designed nursing instructions according to literature review, available resources, and patient assessment needs. It consists of two parts: Part (1): Information about definition, causes, signs and symptoms, diagnostic studies of neutropenia, and medical management. Part (2): teaching the patients the importance and how to improve their self -care behavior (personal hygiene, hand hygiene, dietary hygiene, and oral hygiene) 
The designed nursing instructions were validated by five oncology expert panel members.

\subsection{Procedure}

The study was conducted through the following three phases:

Phase I: Assessment phase

- Review of relevant literature (nursing and medical textbooks, journals, and internet resources about neutropenia, care and patient's outcomes).

- Tools were designed and tested for content validity by 5 experts of medical surgical nursing field and oncology field at Assiut University and tested for reliability by using internal consistency using Cronbach test, the tools proved to be reliable at (0.723).

- An official permission was obtained from the ethical committee of the faculty of nursing and from the Dean of South Egypt Cancer Institute at Assiut university hospital to conduct the study.

- A pilot study was conducted on $10 \%$ of sample (6 patients) in the selected setting to evaluate the applicability and clarity of the used tools. Modifications were done based on the results. Those patients who were involved in the pilot study were excluded from the final study sample.

- $\quad$ Phase II: Implementation phase

- Patients were interviewed by the researcher to initiate line of communication, purpose and nature of the study was explained, then patient's written agreement for voluntary participation was obtained.

- Patient assessment sheet and self-care behavior assessment sheet was filled out from patients participate in the study (tool I and tool II).

- The designed nursing instructions (booklet) were applied, the education session was provided by the researcher in patient's room. Each session took 15-30 minutes. After each session there was 5-10 minutes for discussion and feedback. Reinforcement of teaching was performed according to patient's needs to ensure their understanding. Each patient obtained a copy of the booklet; also the researcher used pictures and diagram for illustration.

- Data were assured confidentiality and anonymity and were collected using the pre-mentioned study tools.

- The tools filled out through interviewing, this study was carried out through the period from (15-1-2017) to (15-6-2017) at morning and afternoon shifts

Phase III: Evaluation phase:During this phase the researcher evaluated the effect of applying the designed nursing instructions on knowledge and self-care behavior among patients with chemotherapy induced neutropenia using (tool I part II), and (tool II) after two weeks.

Statistical design:

The data analysis was carried out using software package computer program SPSS (version, 23) the collected data were tabulated and analyzed by using frequency, percentage distribution, mean and standard deviation. The level of statistically significant was considered at $\mathrm{p}<0.05$.

Limitations of the study:

Six patients did not complete the research because they died.

\section{Results}

Part I: Demographic data of patients

Figure (1): Distribution of demographic data for the studied patients $(\mathrm{n}=60)$

\begin{tabular}{|c|c|c|}
\hline Variables & $\mathrm{N}$. & $\%$ \\
\hline \multicolumn{3}{|l|}{ Type of cancer } \\
\hline - $\quad$ Acute myeloid leukemia & 21 & 35.0 \\
\hline - $\quad$ Cancer ovary & 6 & 10.0 \\
\hline - $\quad$ Ewing's sarcoma & 1 & 1.7 \\
\hline
\end{tabular}

\section{Percentage distrbuation of demographic data}

n Percent

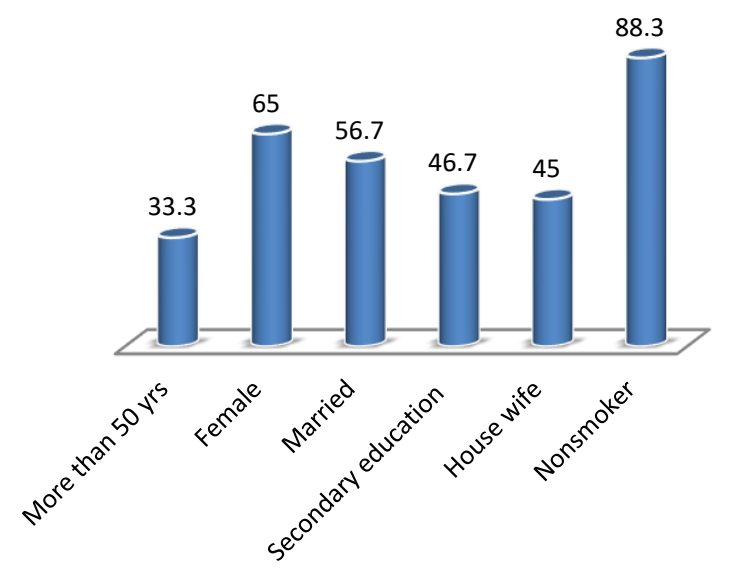

Fig. 1: Illustrated That, More Than Half of the Studied Patients (65\%) Were Female, $(33.3 \%)$ their Age was 50 Years and More, More Than Half $(56.7 \%)$ Were Married. As Regards To Educational Level, the Highest Percentage $(46.7 \%)$ Had Secondary Education. In Relation to Type of Employment the Highest Percentage (45\%) Was Housewife. Regarding Smoking the Majority of Them (88.3\%) Were Non-Smokers (GCSF): Granulocyte Colony Stimulating Factor?

Table (1): Presented that highest percentage of diagnosis (35\%) was Acute Myeloid Leukemia (AML). Regarding presence of chronic illness, the highest percentages complained from cardiovascular disease, In relation to Presence of open wound only (3\%) had an open wound. Regarding medication used more than half of patients $(58.3 \%)$ were using Granulocyte colony stimulating factor (GCSF).

(ECOG): Developed by the Eastern Cooperative Oncology Group Table (2): Presented that more than half of the studied patients $(60$ $\%$ ) located in" Grade 0 " of ECOG performance status which present (Full active, able to carry on all pre-disease performance without restriction).

Part II: knowledge assessment

* Statistically significant difference $(\mathrm{p}<0.05) * *$ highly statistically significant difference $(\mathrm{p}<0.01)$

\{Six patients not completed the research because they died

Table (3): Showes a highly statistically significant difference between the studied patients pre and post application of designed nursing instructions regarding their knowledge.

Part III: self-care behavior assessment

* Statistically significant difference $(\mathrm{p}<0.05) * *$ highly statistically significant difference $(\mathrm{p}<0.01)$

Table (4): Illustrated that there were statistically significant differences between pre and post application of the designed nursing instructions regarding self -care behavior assessment among the studied patients. 


\begin{tabular}{|c|c|c|}
\hline & & \\
\hline - $\quad$ Multiple myeloma & 2 & 3.3 \\
\hline - $\quad$ Acute lymphatic leukemia & 6 & 10.0 \\
\hline - $\quad$ large retroperitoneal mass & 1 & 1.7 \\
\hline - $\quad$ Relapsed all & 4 & 6.7 \\
\hline - Myeloid fibrosis & 1 & 1.7 \\
\hline - $\quad$ Breast cancer & 5 & 8.3 \\
\hline - $\quad$ Cancer colon & 4 & 6.7 \\
\hline - $\quad$ Cancer lung & 3 & 5.0 \\
\hline - $\quad \mathrm{NHL}$ & 4 & 6.7 \\
\hline - $\quad$ Cancer bladder & 2 & 3.3 \\
\hline $\begin{array}{ll}\text { - } & \text { Presence of chronic illness } \\
\text { - } & \text { Liver disease }\end{array}$ & & \\
\hline - $\quad$ Yes & 5 & 8.3 \\
\hline - $\quad$ No & 55 & 91.7 \\
\hline Renal disease & & \\
\hline - $\quad$ Yes & 1 & 1.7 \\
\hline - $\quad$ No & 59 & 98.3 \\
\hline Cardiovascular disease & & \\
\hline - $\quad$ Yes & 12 & 20.0 \\
\hline - $\quad$ No & 48 & 80.0 \\
\hline Presence of open wound & & \\
\hline - $\quad$ Yes & 2 & 3.3 \\
\hline$\bullet \quad$ No & 58 & 96.7 \\
\hline Previous radiation therapy & & \\
\hline - $\quad$ Yes & 0 & 0 \\
\hline - $\quad$ No & 60 & 100.0 \\
\hline Medication used & & \\
\hline Antibiotic & & \\
\hline - $\quad$ Yes & 34 & 56.7 \\
\hline - $\quad$ No & 26 & 43.3 \\
\hline Anti-fungal & & \\
\hline - $\quad$ Yes & 28 & 46.6 \\
\hline$\bullet \quad$ No & 32 & 53.3 \\
\hline Antibiotic and anti-fungal & & \\
\hline - $\quad$ Yes & 26 & 43.3 \\
\hline - $\quad$ No & 34 & 56. \\
\hline G-CSF & & \\
\hline - $\quad$ Yes & 35 & 58.3 \\
\hline - $\quad$ No & 25 & 41.7 \\
\hline
\end{tabular}

Table 2: Percentage Distribution for Medical Data of the Studied Patients Regarding to ECOG Performance Status (N=60)

\begin{tabular}{|c|c|c|c|c|c|c|c|c|c|c|c|c|}
\hline Variable & Gra & & Gra & & Gra & & Gra & & Gra & & Gra & \\
\hline & N. & $\%$ & N. & $\%$ & N. & $\%$ & N. & $\%$ & N. & $\%$ & N. & $\%$ \\
\hline $\begin{array}{l}\text { Full active, able to carry on all pre-dis- } \\
\text { ease performance without restriction. }\end{array}$ & 36 & 60 & - & - & - & - & & & & & & \\
\hline $\begin{array}{l}\text { Restricted in physically strenuous activ- } \\
\text { ity but ambulatory and able to carry out } \\
\text { work of a light or sedentary nature(light } \\
\text { house work, office work) }\end{array}$ & - & - & 10 & 16.7 & & & & & & & & \\
\hline Ambulatory and capable of all self-care & & & & & & & & & & & & \\
\hline $\begin{array}{l}\text { but unable to carry out any work activi- } \\
\text { ties ,up and about more than } 50 \% \text { of wak- } \\
\text { ing hours }\end{array}$ & & & & & 3 & 5.0 & & & & & & \\
\hline $\begin{array}{l}\text { Capable of only limited self -care ,con- } \\
\text { fined to bed or chair more than } 50 \% \text { of } \\
\text { waking hours }\end{array}$ & & & & & & & 8 & 13.3 & & & & \\
\hline $\begin{array}{l}\text { Completely disabled,cannot carry on any } \\
\text { self-care: totally confined to bed or chair }\end{array}$ & & & & & & & & & 3 & 5.0 & & \\
\hline Dead & & & & & & & & & & & 0 & 0 \\
\hline
\end{tabular}

Table 3: Comparison between Total Knowledge Scores Pre and Post Application of the Designed Nursing Instructions

\begin{tabular}{|c|c|c|c|c|c|}
\hline Variable & Pre & & Post & & P.value \\
\hline & N. & $\%$ & N. & $\%$ & $0.001 * *$ \\
\hline - $\quad$ Satisfactory knowledge (More than 50\%) & 5 & 8.3 & 51 & 94.4 & \\
\hline - Unsatisfactory knowledge (Less than 50\%) & 55 & 91.7 & 3 & 5.6 & \\
\hline
\end{tabular}

Table 4: Comparison between Pre, and Post Application of the Designed Nursing Instructions Regarding Self -Care Behavior of the Studied Patients

\begin{tabular}{|c|c|c|c|c|c|c|c|c|c|c|c|c|c|c|c|c|c|}
\hline \multirow[t]{3}{*}{ Variable } & \multicolumn{8}{|c|}{ Pre $(N .=60)$} & \multicolumn{8}{|c|}{ Post $(\mathrm{N} .=54)$} & \multirow{2}{*}{ P.V } \\
\hline & \multicolumn{2}{|c|}{ Never } & \multicolumn{2}{|c|}{ Seldom } & \multicolumn{2}{|c|}{ usually } & \multicolumn{2}{|c|}{ always } & \multicolumn{2}{|c|}{ Never } & \multicolumn{2}{|c|}{ Seldom } & \multicolumn{2}{|c|}{ usually } & \multicolumn{2}{|c|}{ Always } & \\
\hline & N. & $\%$ & $\mathrm{~N}$. & $\%$ & $\mathrm{~N}$. & $\%$ & $\mathrm{~N}$. & $\%$ & $\mathrm{~N}$. & $\%$ & $\mathrm{~N}$. & $\%$ & $\mathrm{~N}$. & $\%$ & $\mathrm{~N}$. & $\%$ & \\
\hline $\begin{array}{l}\text { personal hy- } \\
\text { giene }\end{array}$ & 22 & 36.7 & 38 & 63.3 & 0 & 0 & 0 & 0 & 2 & 3.7 & 1 & 1.85 & 6 & 11.1 & 45 & 83.3 & $0.001 * *$ \\
\hline Hand hygiene & 15 & 25.0 & 44 & 73.3 & 1 & 1.7 & 0 & 0 & 0 & 0 & 0 & 0 & 5 & 9.25 & 49 & 90.7 & $0.001 * *$ \\
\hline $\begin{array}{l}\text { dietary hy- } \\
\text { giene }\end{array}$ & 25 & 41.7 & 35 & 58.3 & 0 & 0 & 0 & 0 & 39 & 72.2 & 11 & 20.4 & 3 & 5.55 & 1 & 1.85 & $0.02 * *$ \\
\hline Oral hygiene & 24 & 40.0 & 36 & 60.0 & 0 & 0 & 0 & 0 & 0 & 0 & 0 & 0 & 7 & 12.96 & 47 & 87.0 & $0.001 * *$ \\
\hline
\end{tabular}


Table 5: Relation between Patient's Knowledge about Neutropenic and their Self-Care Behavior

\begin{tabular}{|c|c|c|c|c|c|c|c|c|c|}
\hline \multirow[t]{4}{*}{ Variable } & \multicolumn{8}{|c|}{ Knowledge } & \multirow[t]{2}{*}{ P.v } \\
\hline & \multicolumn{4}{|c|}{ Pre } & \multicolumn{4}{|c|}{ Post } & \\
\hline & & & Un & tory & Sat & ory & & tory & \multirow{4}{*}{$0.00 *$} \\
\hline & $\mathrm{N}$. & $\%$ & $\mathrm{~N}$. & $\%$ & $\mathrm{~N}$. & $\%$ & $\mathrm{~N}$. & $\%$ & \\
\hline Positive self-care behavior & 0 & 0 & 0 & 0 & 51 & 94.4 & 3 & 5.55 & \\
\hline Negative self-care behavior & 5 & 8.3 & 55 & 91.7 & 0 & 0 & 0 & 0 & \\
\hline
\end{tabular}

* Statistically significant difference $(\mathrm{p}<0.05) * *$ highly statistically significant difference $(\mathrm{p}<0.01)$.

Table (5): Shows a statistically significant difference between patient's level of knowledge and their self-care behavior pre and post application of the designed nursing instructions

\section{Discussion}

The discussion of this study was presented in the following sequence: part I described demographic characteristics and medical data, part II presented the patient's level of knowledge about neutropenia pre and post applying the designed nursing instructions, and part III presented the effect of the designed nursing instructions on self -care behavior among patients with chemotherapy induced neutropenia.

\subsection{Part I: demographic characteristics and medical data}

Based on the results of the present study, more than half of the studied patients were female, married and their age was 50 years and more, this agree with Schelenz et al. (2012) who demonstrated the variability of the incidence of febrile neutropenia according to several factors including cancer type, chemotherapy regimen, antibiotic treatment, age and sex. Regarding to the age and sex, the mean age was 63 years (two- thirds were at least 60 years old) and more than half of patients was female.

Regarding marital status, the present study revealed that more than half of the studied group was married, in relation to type of employment the highest percentage was housewife. As regards to educational level, the highest percentage had secondary education. These results were in agreement with Mak and Ching (2015) who studied the effect of an educational program for breast cancer patients on knowledge, self-care behavior and hand washing competence on prevention of febrile neutropenia, they reported that most of the patients in the study were married, educated to primary and secondary school level and housewife.

- The results of the present study showed that the majority of the studied patients were non -smokers and this was in the same line with O'Malley et al. (2013) who reported that non- smokers had increased neutropenia versus current smokers with different tumor types.

The current study revealed that the highest percentage of diagnosis was Acute Myeloid Leukemia (AML) and this came in line with Dulisse et al. (2013) who reported that high incidence of febrile neutropenia may be clarified by the higher prevalence of underlying hematological malignancies that increase the risk for febrile neutropenia, as $10-50 \%$ of patients with solid tumors may develop Febrile neutropenia, compared with $80 \%$ of those with hematological malignancies.

The result of the current study noticed the presence of chronic illness with patients complained from neutropenia and most of these illnesses were cardiovascular disease and this was in line with Kuderer et al. (2004) who revealed that the presence of comorbid conditions with cancer has been shown to increase the risk for neutropenia and its complications, heart disease and renal disease have been revealed to increase the risk for febrile neutropenia.

As regard medication use with neutropenia the present study revealed that more than half of the studied group used antibiotics and Granulocyte colony-stimulating factors (G-CSF) as a treatment at the onset of admission and this agreed with Mohamed et al. (2016) who studied: Risks and consequences of chemotherapy-induced neutropenia patients in Egypt and they reported that therapy with G-CSF was associated with faster bone marrow recovery, with a significant negative correlation between duration of neutropenia and G-CSF (r-0.37, P $\leq 0.001)$.

Regarding ECOG performance status which developed by (the Eastern Cooperative Oncology Group), the current study revealed that more than half of the studied patients located in grade " 0 " full active, able to carry on all pre disease performance without restriction, this finding disagree with Voog et al. (2000) who reported that three studies have shown that, in addition to age, poor performance status is a significant risk factor for chemotherapy- induced neutropenia. Our findings in this study were similar to the National Comprehensive Cancer Network guideline update (2011), when they showed that poor performance status was not clearly identified as a risk factor for neutropenia.

\subsection{Patient knowledge about neutropenia}

The current study revealed a lack in patients' knowledge related to chemotherapy induced neutropenia (CIN) at baseline where most of them had unsatisfactory knowledge levels. However, post applying the designed nursing instructions the studied patients achieved significant improvements in knowledge. This result agrees with Finkler et al. (2003) who issued a survey to 79 oncology patients to assess their knowledge and understanding of chemotherapy induced neutropenia (CIN) and their results showed that more than half of patients did not know when to contact their doctor or oncology nurse and approximately one-third of their patients did not know the signs and symptoms of infection and in addition around half of them could not describe self-care measures.

The current study revealed that use of teaching material during application of designed nursing instructions improved patient's level of knowledge, and this in line with Kinnane and Thompson.(2008) who concluded that patients who viewed the video in conjunction with standard chemotherapy education improve retaining of information regarding management of predictable chemotherapy side effects and reporting of treatment-related symptoms.

From the researcher opinion it has necessary for patients to play a role in the early detection of chemotherapy induced neutropenia (CIN) in order to prevent the development of life-threatening infections, a patient's role, however, is largely dependent upon the information they receive from oncology nurses or physicians.

\subsection{Self-care behavior of the studied patients}

The current study revealed that the majority of the studied patients were having negative self-care behavior score levels in relation to hand hygiene and oral hygiene, dietary hygiene and personal hygiene before application of the designed nursing instructions, while after applying the designed nursing instructions they showed positive self-care behavior scores in most items.

Mak and Ching (2015) in there study reported that self-care behaviors had increased in intervention group than control group. Wilson (1997) also in the same line reported that, matching instructional methods to individual readiness to learn, learning style, and learning speed are generally regarded as desirable and more likely to be effective in changing patient's behavior. In contrast, audio - or videotape patient education program even typically designed for delivery to individual patients is inflexible across individual differences. Also the current results comes in line with Berman et al. (2009) who reported that, hand washing is the best way to discontinue the 
spread of infections. In addition to Kearney et al. (2006) who revealed that, frequent oral care is very important and most effective intervention to avoid oral mucositis from chemotherapy. It is therefore important for the nurse to teach the patients about oral hygiene technique and the importance to comply with it, also to teach patients the role of the neutropenic diet in preventing infections in patients receiving chemotherapy as low-microbial diet for neutropenic patients reduces the potential for infection associated with bacterial translocation.

Regarding relation between knowledge and self-care behaviors the present study showed that there was a statistically significant difference between patient's level of knowledge and their self -care behavior pre and post application of the designed nursing instructions, this result supported with Prutipinyo et al. (2012) who concluded that knowledge is associated with self-care behaviors of cancer patients who received chemotherapy, and added providing health promotion for patients is necessary for them to have better self-care behaviors in their study about "self-care behaviors of chemotherapy patients".

\section{Conclusion\& recommendations}

Based on the results of the present study, it can be concluded that there were highly statistically significant differences between "pre and post" application of the designed nursing instructions regarding knowledge and self-care behavior for neutropenic patients undergoing chemotherapy.We can recommended that:

- Each neutropenic patient should be given a booklet including knowledge about neutropenia, and how to practice standard self -care behavior.

- Education of patients, families, and healthcare workers, are keys to prompt recognition about potentially life-threatening symptoms associated with neutropenia.

\section{References}

[1] Akhil M. Neutropenia in the newborn, Current Opinion in Hematology. (2014) PP: 43-49.

[2] Artinian, N. T., Magnan, M., Sloan, M., \& Lange, M. P. Self-care behaviors among patients with heart failure. Heart \& Lung: The Journal of Acute andCritical Care. (2002); 31(3), 161-172.

[3] Badr M., Hassan T., Sakr H., Karam N., Abdel Rahman D., Shahbah D., Zakaria M.,and Fehr S .Chemotherapy-induced neutropenia among pediatric cancer patients in Egypt: Risks and consequences. Published online on (2016); July 12, 2016 https://doi.org/10.3892/mco.2016.957.

[4] Bastable SB., Grambet P., Jacobs K. and Sopczyk DL. Health professionals as educator: Principles of teaching and learning. Sudbury, MA: Jones \& Bartlett Learning, LLC. ., (2011); PP: 6.

[5] Berman A., Snyder S. and Kozier Guidelines for Hand Hygiene in Health Care Settings: Recommendations of the Healthcare Infection Control Practices Advisory Committee and... Philadelphia Elsevier Saunders. (2009)

[6] Dulisse B, Li X, Gayle JA, Barron RL, Ernst FR, Rothman KJ, Legg JC and Kaye JA .A retrospective study of the clinical and economic burden during hospitalizations among cancer patients with febrile neutropenia. J Med Econ. (2013); 16:720-735 https://doi.org/10.3111/13696998.2013.782034 ...

[7] Finkler J., Rapp C. and Johnston M. Neutropenia in oncology patients, standardizing an educational approach and measuring outcomes. Proceedings from the 2003 annual congress of the Oncology Nursing Society.

[8] Hawley EL., Loney M. and Wiece M. Development of tools and processes to improve treatment times in patients with febrile neutropenia. Clinical Journal of Oncology Nursing. , (2011); PP: 15: $53-$ 56.

[9] Jaarsma, T., \& Van Veldhuisen, D. J. When, how and where should we "coach"patients with heart failure: The coach results in perspective. European Journal Heart Failure. (2008); 10(4), 331-333. https://doi.org/10.1016/j.ejheart.2008.02.017.

[10] Kearney N., Richardaon A. and Foubert J. Oral care protocol for patient in chemotherapy. Nursing patient with cancer, principle and practice. Second ed., Churchill Living stone. (2006) PP: 585.

[11] Kinnane N and Thompson L. Evaluation of the addition of videobased education for patients receiving standard pre-chemotherapy education. Eur J Cancer Care (Engl) (2008); 17:328-39. https://doi.org/10.1111/j.1365-2354.2007.00846.x.

[12] Kuderer NM, Dale D. and Crawford J. The impact of febrile neutropenia in hospitalized cancer patients: morbidity, mortality, and cost. Proc Am Soc Clin Oncol. (2004); PP: 23:529.

[13] Loerzel, V. Fever with neutropenia. In M. Hickey \& S. Newton (Eds.), Telephone triage for oncology nurses. Pittsburgh, PA: Oncology Nursing Society. (2005); PP125-127.

[14] National Comprehensive Cancer Network.Clinical Practice Guidelines in Oncology, Myeloid Growth Factors. (2011); vol(1).

[15] O’Malley M., Healy P., Daignault S. and Ramnath N. Cigarette Smoking and Gemcitabine-Induced Neutropenia in Advanced Solid Tumors. (2013) vol (85) issue (4) PP216-222.

[16] Oken MM. Creech RH., Tormey DC., Horton J., Davis TE, and McFadden ET., Toxicity and response criteria of the Eastern Cooperative Oncology Group.Am J Clin Oncol. (1982); PP: 5:649-55

[17] Polit DF, Beck CT. and Owen SV. Is the CVI an acceptable indicator of content validity? Appraisal and recommendations. Res Nurs Health. (2007); PP30:459-67.

[18] Prutipinyo C., Maikeow K., and Sirichotiratana N. Self-care behaviors of chemotherapy patients. J Med Assoc Thai. (2012); Jun; 95 Suppl 6:S30-7.

[19] Ropka ME and Padilla G. Assessment of neutropenia-related quality of life in a clinical setting, Oncol Nurs Forum. (2007); PP: 34: 403409.

[20] Schelenz S., Giles D. and Abdallah S.Epidemiology, management and economic impact of febrile neutropenia in oncology patients receiving routine care at a regional UK cancer centre. (2012) Ann Oncol vol( 23) issue (7) PP 1889-1893.

[21] Villafuerte GP., Villalon L., Losa JE. and Henriquez CC. Treatment of Febrile Neutropenia and Prophylaxis in Hematologic Malignancies, A Critical Review and Update, Advances in Hematology. (2014); PP: 9.

[22] Voog E., Bienvenu J. and Warzocha K. Factors that predict chemotherapy-induced myelosuppression in lymphoma patients: role of the tumor necrosis factor ligand-receptor sys- tem. J Clin Oncol. (2000); PP: 18:325-331

[23] Mak WC and Ching SS. Effect of an education program on knowledge, self-care behavior and hand washing competence on prevention of febrile neutropenia among breast cancer patients receiving Doxorubicin and Cyclophosphamide in Chemotherapy Day Centre Asia Pac J Oncol Nurs. (2015); Oct-Dec; 2(4): 276-288.

[24] Weycker D., Barron R., Kartashov A., Legg J. and Lyman GH. Incidence, treatment, and consequences of chemotherapy-induced febrile neutropenia in the inpatient and outpatient settings. J Oncol Pharm Pract. (2014); PP: 20: 190-198.

[25] Wilson SR.Individual versus group education: Is one better? Patien Educ Couns. (1997) 321(Supp 1):S67-75. https://doi.org/10.1016/S0738-3991(97)00098-0. 